\title{
CLIQUES AND COLORINGS IN GENERALIZED PALEY GRAPHS AND AN APPROACH TO SYNCHRONIZATION
}

\author{
CSABA SCHNEIDER AND ANA SILVA
}

\begin{abstract}
Given a finite field, one can form a directed graph using the field elements as vertices and connecting two vertices if their difference lies in a fixed subgroup of the multiplicative group. If -1 is contained in this fixed subgroup, then we obtain an undirected graph that is referred to as a generalized Paley graph. In this paper we study generalized Paley graphs whose clique and chromatic numbers coincide and link this theory to the study of the synchronization property in 1-dimensional primitive affine permutation groups.
\end{abstract}

\section{INTRODUCTION}

The synchronization property for permutation groups emerged from problems related to the Černý conjecture [Čer64] in the theory of finite state automata and was first independently defined by João Araújo and Benjamin Steinberg; see [A08, AS06. As was shown by Arnold and Steinberg AS06, a synchronizing group is always primitive. However, the converse is not true, which makes it interesting to study the problem: Which primitive groups are synchronizing? Neumann Neu09. proved several basic properties of synchronizing groups, and he also observed that a primitive group that can be embedded into a wreath product in product action is always non-synchronizing. This shows, in the terminology of Cameron [Cam99], that a synchronizing group must be a basic primitive group. Therefore the O'Nan-Scott type of a synchronizing group is almost simple, affine, or simple diagonal. The study of precisely which groups in these classes are synchronizing leads to deep problems in combinatorics, graph theory, and finite geometry. For instance, deciding whether a classical group in its action on the associated polar space is synchronizing requires a study of spreads and ovoids of the polar space; see Cam10, Theorem 9 of Part 6].

In this paper we study which of the members of a particular class of affine groups are synchronizing. These groups are primitive subgroups of the 1-dimensional affine groups $\operatorname{AGL}(1, q)$ acting on the set $\mathbb{F}_{q}$ for odd prime-powers $q$. Neumann [Neu09] introduced a graph theoretic characterization of non-synchronizing groups in terms of cliques and colorings of their generalized undirected orbital graphs. The undirected orbital graphs of the groups we study are isomorphic to certain 
graphs that can be considered as generalizations of the Paley graphs of finite fields, first defined in Pal33. Such graphs were also considered in [LP09]. Hence, using Neumann's characterization of nonsynchronizing groups (Lemma 2.3), the study of the synchonization property in such affine groups leads to an investigation of the clique and chromatic numbers of generalized Paley graphs.

Calculating the clique and the chromatic numbers can be notoriously difficult already for Paley graphs as shown by [BDR88]; see also Mik] for a recent discussion. Hence our results are far from conclusive. Nevertheless, we prove some theorems that describe situations when the chromatic and the clique numbers of generalized Paley graphs are equal (Theorem 4.7), and we apply these results to determine if certain 1dimensional affine groups are synchronizing (Theorem 5.2).

In Section 2, we give a brief summary of synchronizing groups. In Section 3, we link affine synchronizing groups to generalized Paley graphs. In Section 4, we prove our main result (Theorem 4.7) concerning the clique and chromatic numbers of generalized Paley graphs that state several sufficient or necessary conditions for these numbers to be equal. Finally, in Section 5, we apply Theorem 4.7 in the study of synchronization in 1-dimensional affine groups.

Acknowledgment. The research that led to this paper was carried out as part of the second author's MSc project in the Center of Algebra of the University of Lisbon. We both are grateful to the Center for supporting our work.

\section{Synchronizing Groups}

Let $\Omega$ be a finite set and let $\mathcal{P}$ be a partition of $\Omega$. A subset $S$ of $\Omega$ is called a section for $\mathcal{P}$ if $S$ contains precisely one element from each part of $\mathcal{P}$. Let $G \leqslant \operatorname{Sym}(\Omega)$. If $\mathcal{P}$ admits a section $S$ such that $S g$ is a section for all $g \in G$ then $\mathcal{P}$ is said to be a section-regular partition for $G$ or a $G$-regular partition. In this case we say that the section $S$ witnesses the $G$-regularity of $\mathcal{P}$.

The partitions $\{\Omega\}$ and $\{\{\omega\} \mid \omega \in \Omega\}$ are clearly $G$-regular for any permutation group $G$ acting on $\Omega$. These partitions are said to be trivial. A permutation group $G$ acting on a set $\Omega$ is called synchronizing if $G \neq 1$ and there are no non-trivial $G$-regular partitions of $\Omega$. It follows that synchronizing groups are transitive and primitive, since a $G$-invariant partition is clearly $G$-regular. Furthermore, it is not hard to see that 2-homogeneous groups are synchronizing.

The following lemma summarizes the basic properties of $G$-regular partitions for transitive groups; see [Neu09, Section 2].

Lemma 2.1. Let $G$ be a transitive group acting on a set $\Omega$.

(1) A G-regular partition is uniform, that is, all its parts have the same size. 
(2) If $G$ is a primitive group and $\mathcal{P}$ is a non-trivial $G$-regular partition, then $|\mathcal{P}| \geqslant 3$ and $|P| \geqslant 3$ for all $P \in \mathcal{P}$.

As we mentioned in the introduction, Neumann [Neu09] showed that a primitive group that can be embedded into a wreath product in product action is non-synchronizing. In the following lemma we rephrase this result in the language of cartesian decompositions, introduced in PBS03]. A cartesian decomposition of a set $\Omega$ is a set $\Sigma=\left\{\mathcal{P}_{1}, \ldots, \mathcal{P}_{t}\right\}$ of non-trivial partitions of $\Omega$ such that

$$
\left|P_{1} \cap \cdots \cap P_{t}\right|=1 \text { for all } P_{1} \in \mathcal{P}_{1}, \ldots, P_{t} \in \mathcal{P}_{t} .
$$

Lemma 2.2. Let $G$ be a primitive group acting on a finite set $\Omega$. If $G$ preserves a cartesian decomposition of $\Omega$, then $G$ is non-synchronizing.

Proof. Let $\Sigma=\left\{\mathcal{P}_{1}, \ldots, \mathcal{P}_{t}\right\}$ be a cartesian decomposition of $\Omega$ which is invariant under $G$. We start by showing that the group $G$ must be transitive on $\Sigma$. Assume by contradiction that $G$ is intransitive on $\Sigma$. Then we may assume without loss of generality that $\Sigma_{1}=\left\{\mathcal{P}_{1}, \ldots, \mathcal{P}_{s}\right\}$, with $s<t$, is a $G$-orbit.

Let $P_{i} \in \mathcal{P}_{i}$, for $i \in\{1, \ldots, s\}$, and set $B=P_{1} \cap \cdots \cap P_{s}$. We claim that $B$ is a non-trivial block for $G$. Since the partitions $\mathcal{P}_{j}$ are nontrivial for all $j \in\{1, \ldots, t\}$, we have that $B \neq \Omega$. Next, we show that $|B|>1$. Let $P_{r}, P_{r}^{\prime} \in \mathcal{P}_{r}$ with $r>s$ and such that $P_{r} \neq P_{r}^{\prime}$. Then, since $\Sigma$ is a cartesian decomposition of $\Omega$, it follows that $B \cap P_{r} \neq \emptyset$ and $B \cap P_{r}^{\prime} \neq \emptyset$. As $P_{r} \cap P_{r}^{\prime}=\emptyset$, we obtain $B$ contains at least two elements, as was claimed. Now let $g \in G$. If $\left\{P_{1} g, \ldots, P_{s} g\right\}=\left\{P_{1}, \ldots, P_{s}\right\}$, then $B g=B$, while otherwise, $B g \cap B=\emptyset$. Thus $B$ is non-trivial a block for $G$, which is a contradiction since we assume that $G$ is primitive. Hence $G$ is transitive on the cartesian decomposition $\Sigma$.

Next, we show that $\mathcal{P}_{1}$ is a $G$-regular partition of $\Omega$. By the first part of the proof, $G$ is transitive on $\Sigma$. Then $\left|\mathcal{P}_{i}\right|=\left|\mathcal{P}_{j}\right|$ for all $\mathcal{P}_{i}, \mathcal{P}_{j} \in \Sigma$. For all $i \in\{1, \ldots, t\}$, fix a bijection $\alpha_{i}: \mathcal{P}_{1} \rightarrow \mathcal{P}_{i}$. Define, for $P \in \mathcal{P}_{1}$ the element $\omega_{P} \in \Omega$ to be the unique element in $P \cap P \alpha_{2} \cap \cdots \cap P \alpha_{t}$. Set $S=\left\{\omega_{P}: P \in \mathcal{P}_{1}\right\}$. Note that $S$ is a section for all $\mathcal{P}_{i} \in \Sigma$. Thus if $g \in G$, then $S$ is a section for $\mathcal{P}_{1} g^{-1}$, which gives that $S g$ is a section for $\mathcal{P}_{1}$. Therefore $S$ witnesses the section-regular property for $\mathcal{P}_{1}$ and thus $\mathcal{P}_{1}$ is a non-trivial $G$-regular partition. Hence $G$ is a non-synchronizing group.

Neumann introduced in [Neu09] a graph theoretic characterization of synchronizing groups. If $G$ is a permutation group acting on $\Omega$ then the $G$-action on $\Omega$ can naturally be extended to the sets

$$
\begin{aligned}
\Omega^{2} & =\{(\alpha, \beta) \mid \alpha, \beta \in \Omega\} \text { and } \\
\Omega^{\{2\}} & =\{\{\alpha, \beta\} \mid \alpha, \beta \in \Omega \text { with } \alpha \neq \beta\} .
\end{aligned}
$$

Suppose that $G$ is transitive on $\Omega$. The $G$-orbits in $\Omega^{2}$ are called orbitals and we will refer to the $G$-orbits on $\Omega^{\{2\}}$ as undirected orbitals. 
For any subset $\Delta$ of $\Omega^{2}$, the pair $(\Omega, \Delta)$ is a directed graph, while for such a subset $\Delta$ in $\Omega^{\{2\}}$, the pair $(\Omega, \Delta)$ is an undirected simple graph. In this paper the word 'graph' will mean an underected simple graph. If we refer to a directed graph, then we will write 'directed graph'. Such a graph or directed graph admits $G$ as a group of automorphisms if and only if $\Delta$ is $G$-invariant; that is, it is a union of orbitals or undirected orbitals.

A complete subgraph of a graph $\Gamma$ is called a clique. The largest among the cardinalities of the cliques of a graph is said to be the clique number. An empty subgraph of $\Gamma$ is said to be an independent set and the largest among the cardinalities of the independent sets is the independence number. The chromatic number is the smallest number of colors necessary to color $\Gamma$ such that adjacent vertices receive different colors.

Lemma 2.3 (Theorem 10 of Chapter 5 [Cam10]). A transitive group $G$ acting on $\Omega$ is non-synchronizing if and only if there is a $G$-invariant subset $\Delta$ of $\Omega^{\{2\}}$ such that the chromatic number of the undirected graph $(\Omega, \Delta)$ is equal to its clique number.

We will characterize the undirected orbitals of 1-dimensional affine groups in Lemma 3.2 using the following connection between orbitals and suborbits; see [DM96, Section 3.2]. Suppose that $G$ is a transitive group acting on $\Omega$. For a fixed $\alpha \in \Omega$, the correspondence $\Delta \mapsto \Delta(\alpha)$ where $\Delta(\alpha)=\{\beta \mid(\alpha, \beta) \in \Delta\}$ is a bijection between the set of $G$ orbitals and the set of orbits of the stabilizer $G_{\alpha}$. The orbits of the stabilizer $G_{\alpha}$ are referred to as suborbits. If $\Delta$ is an orbital, then so is the set $\Delta^{\prime}=\{(\beta, \gamma) \mid(\gamma, \beta) \in \Delta\}$, and $\Delta^{\prime}$ is called the paired orbital of $\Delta$. In this case we will also say that the suborbit $\Delta^{\prime}(\alpha)$ is the paired suborbit of $\Delta(\alpha)$. The orbital $\{(\beta, \beta) \mid \beta \in \Omega\}$ is referred to as the diagonal orbital. The undirected orbitals are in one-to-one correspondence with the set of sets $\left\{\Delta, \Delta^{\prime}\right\}$ where $\Delta$ and $\Delta^{\prime}$ are non-diagonal paired orbitals (such a set can have one or two elements depending on whether $\left.\Delta^{\prime}=\Delta\right)$, and hence they are also in one-to-one correspondence with the set of sets $\left\{\Delta(\alpha), \Delta^{\prime}(\alpha)\right\}$ where $\Delta(\alpha)$ and $\Delta^{\prime}(\alpha)$ are paired suborbits that are not equal to $\{\alpha\}$.

\section{Generalized Paley graphs as undirected orbital GRAPHS}

The objective of this paper is to study which of the subgroups of the 1-dimensional affine general linear groups are synchronizing. These permutation groups can be constructed as follows. Let $q$ be an odd prime-power and let $m$ be a divisor of $q-1$. We let $\mathbb{F}_{q}$ denote the field of $q$ elements and also let $\mathbb{F}_{q}^{*}$ denote the multiplicative group $\mathbb{F}_{q} \backslash\{0\}$. Set $S_{q, m}=\left\{\alpha^{m} \mid \alpha \in \mathbb{F}_{q}^{*}\right\}$. Then $S_{q, m}$ is a subgroup of $\mathbb{F}_{q}^{*}$ with order $(q-1) / m$. For an element $\alpha \in \mathbb{F}_{q}$, let $\sigma_{\alpha}$ denote the translation map 
$\sigma_{\alpha}: \beta \mapsto \beta+\alpha$ for all $\beta \in \mathbb{F}_{q}$. If $\alpha \in \mathbb{F}_{q}^{*}$, then define the multiplication map $\mu_{\alpha}$ as $\mu_{\alpha}: \beta \mapsto \beta \alpha$ for all $\beta \in \mathbb{F}_{q}$. Then $\sigma_{\alpha}$ and, for $\alpha \in \mathbb{F}_{q}^{*}$, $\mu_{\alpha}$ are elements of $\operatorname{Sym}\left(\mathbb{F}_{q}\right)$. We let $T$ denote the set of elements $\sigma_{\alpha}$ with $\alpha \in \mathbb{F}_{q}$ and $Z_{q, m}$ denote the set of elements $\mu_{\alpha}$ with $\alpha \in S_{q, m}$. Then $T$ and $Z_{q, m}$ are subgroups of $\operatorname{Sym}\left(\mathbb{F}_{q}\right)$ and it is well known that $\left\langle T, Z_{q, m}\right\rangle=T \rtimes Z_{q, m}$. We set $G_{q, m}$ to be $T \rtimes Z_{q, m}$. We consider $G_{q, m}$ as a permutation group of degree $q$ acting on $\mathbb{F}_{q}$. Then $G_{q, 1}=\operatorname{AGL}(1, q)$, and hence $G_{q, m}$ is a transitive subgroup of $\operatorname{AGL}(1, q)$ containing $T$.

Lemma 3.1. Suppose that $q$ and $m$ are as above and suppose that $q=p^{d}$ where $p$ is a prime. Set $r=(q-1) / m$. Then $G_{q, m}$ is a primitive permutation group if and only if $r \nmid p^{i}-1$ for all $1 \leqslant i \leqslant d-1$.

Proof. The group $G_{q, m}$ is primitive if and only if the stabilizer $Z_{q, m}$ of 0 is irreducible considered as a subgroup of $\mathrm{GL}(d, p)$ [DM96, Section 4.7]. As $\left|Z_{q, m}\right|=r$, this happens if and only if $r \nmid p^{i}-1$ for all $i \in\{1, \ldots, d-1\}$ (see [Sho92, Section 2.3]).

In order to study whether the groups $G_{q, m}$ are synchronizing, we use Lemma 2.3, and hence we need first describe the undirected orbitals of $G_{q, m}$ where $m$ is a divisor of $q-1$ as above. Set $r=(q-1) / m$ and set

$$
(\bar{r}, \bar{m})=\left\{\begin{array}{l}
(r, m) \text { if } r \text { is even; } \\
(2 r, m / 2) \text { if } r \text { is odd } .
\end{array}\right.
$$

Thus $\bar{r}$ is always even and $\bar{r} \bar{m}=q-1$. Let $\gamma$ be a primitive element of $\mathbb{F}_{q}$. Then we define, for $i \in\{0, \ldots, \bar{m}-1\}$,

$$
\Delta_{i}=\left\{\{\alpha, \beta\} \mid \alpha-\beta \in S_{q, \bar{m}} \gamma^{i}\right\} \text {. }
$$

As $\bar{r}$ is even, $-1 \in S_{q, \bar{m}}$, and so the pairs $\left(\mathbb{F}_{q}, \Delta_{i}\right)$ define undirected graphs with vertex sets $\mathbb{F}_{q}$. Let $\Gamma_{i}$ denote the graph $\left(\mathbb{F}_{q}, \Delta_{i}\right)$.

To construct the other main object of this paper, consider now an odd prime-power $q$ as above, and let $m$ be a natural number such that $2 m \mid q-1$. We define an undirected graph $\Gamma_{q, m}$ as follows. The vertex set of $\Gamma_{q, m}$ is $\mathbb{F}_{q}$ and its edge set is

$$
\left\{\{\alpha, \beta\} \mid \alpha-\beta \in S_{q, m}\right\} .
$$

The fact that $2 m \mid(q-1)$ guarantees that $-1 \in S_{q, m}$, and so the edge set of $\Gamma_{q, m}$ is well-defined. The graph $\Gamma_{q, m}$ is called a generalized Paley graph for the field $\mathbb{F}_{q}$.

Lemma 3.2. Using the notation above, the following is valid.

(1) $\Delta_{0}, \cdots, \Delta_{\bar{m}-1}$ are precisely the undirected $G_{q, m}$-orbitals.

(2) $\Gamma_{i} \cong \Gamma_{j}$ for all $i, j \in\{0, \ldots, \bar{m}-1\}$;

(3) $\Gamma_{0}=\Gamma_{q, \bar{m}}$.

Proof. As described after Lemma 2.3 , there is a bijection between the set of undirected orbitals of $G_{q, m}$ and the unions of paired suborbits of 
$G_{q, m}$ with respect to the element 0 whose stabilizer is $Z_{q, m}$. These suborbits of $G_{q, m}$ are, in addition to the trivial suborbit $\{0\}$, precisely the cosets $S_{q, m} \gamma^{i}$ with $i \in\{0, \ldots, m-1\}$. The orbital that corresponds to the suborbit $S_{q, m} \gamma^{i}$ is the $G_{q, m}$-orbit of $\left(0, \gamma^{i}\right)$. Its paired orbital is the $G_{q, m^{-}}$-orbit of $\left(\gamma^{i}, 0\right)$. Now $\sigma_{-\gamma^{i}} \in G_{q, m}$, which gives that the $G_{q, m^{-}}$orbit of $\left(\gamma^{i}, 0\right)$ is equal to the $G_{q, m^{-}}$orbit of $\left(\gamma^{i}, 0\right) \sigma_{-\gamma^{i}}=\left(0,-\gamma^{i}\right)$. Thus the paired suborbit of $S_{q, m} \gamma^{i}$ is $-S_{q, m} \gamma^{i}$. The union of $S_{q, m} \gamma^{i}$ and $-S_{q, m} \gamma^{i}$ is equal to $S_{q, \bar{m}} \gamma^{i}$. Thus the undirected orbitals of $G_{q, m}$ are in one-toone correspondence with the cosets $S_{q, \bar{m}} \gamma^{i}$ where $i \in\{0, \ldots, \bar{m}-1\}$. As $G_{q, m}=Z_{q, m} T$, where $T$ is the group of translations $\sigma_{\alpha}$ with $\alpha \in \mathbb{F}_{q}$, for such a coset $S_{q, \bar{m}} \gamma^{i}$, the corresponding undirected orbital is

$$
\begin{aligned}
& \left\{\{0, \beta\} \sigma_{\alpha} \mid \beta \in S_{q, \bar{m}} \gamma^{i} \text { and } \alpha \in \mathbb{F}_{q}\right\}= \\
& \left\{\{\alpha, \beta+\alpha\} \mid \beta \in S_{q, \bar{m}} \gamma^{i} \text { and } \alpha \in \mathbb{F}_{q}\right\}= \\
& \left\{\{\alpha, \beta\} \mid \alpha-\beta \in S_{q, \bar{m}} \gamma^{i}\right\}=\Delta_{i} .
\end{aligned}
$$

This shows assertion (1).

(2) By the definition of the sets $\Delta_{i}$, we have that $\Delta_{0} \gamma^{i}=\Delta_{i}$. Hence $\mu_{\gamma^{i}}$ is an isomorphism between the graphs $\Gamma_{0}$ and $\Gamma_{i}$ for all $i \in\{0, \ldots, \bar{m}-1\}$.

(3) This follows from the definitions of $\Delta_{0}$ and the graph $\Gamma_{q, \bar{m}}$.

For a group $G$ and a subset $C$ such that $C^{-1}=C$ and $1 \notin C$, the Cayley graph Cay $(G, C)$ is defined as the graph with vertex set $G$ and in which $g$ and $h$ are connected if and only if $g h^{-1} \in C$. A Cayley graph Cay $(G, C)$ is said to be normal if $C$ is closed under conjugation by elements of $G$. Clearly, if $G$ is abelian, then all Cayley graphs of $G$ are normal. A graph is said to be regular if every vertex has the same number of neighbours. In this case, the number of neighbours of a vertex is denoted by $\partial(\Gamma)$. For a graph $\Gamma=(\Omega, E)$, we let $\bar{\Gamma}$ denote the complement graph defined by $\left(\Omega, \Omega^{\{2\}} \backslash E\right)$.

Corollary 3.3. Let $q$ be an odd prime-power and $m \geqslant 2$ be such that $2 m \mid q-1$.

(1) The graph $\Gamma_{q, m}$ is vertex-transitive and edge transitive.

(2) We have that $\Gamma_{q, m}$ is a regular graph and $\partial\left(\Gamma_{q, m}\right)=(q-1) / m$.

(3) $\Gamma_{q, m}$ is isomorphic to a subgraph of its complement graph $\overline{\Gamma_{q, m}}$.

(4) We have that $\Gamma_{q, m}=\operatorname{Cay}\left(\mathbb{F}_{q}, S_{q, m}\right)$, viewing $\mathbb{F}_{q}$ as an additive group, and hence $\Gamma_{q, m}$ is a normal Cayley graph.

Proof. As, by Lemma $3.2(3), \Gamma_{q, m}=\left(\mathbb{F}_{q}, \Delta_{0}\right)$ and $\left(\mathbb{F}_{q}, \Delta_{0}\right)$ is vertexand edge-transitive, we obtain statement (1). The fact that $\Gamma_{q, m}$ is regular is a consequence of (1). Further, by the definition of $\Gamma_{q, m}$, the set $S_{q, m}$ coincides with the set of neighbours of the vertex 0 . Thus $\partial \Gamma=\left|S_{q, m}\right|=(q-1) / m$. To show statement (3), we note that the multiplication map $\mu_{\gamma}$, where $\gamma$ is a primitive element of $\mathbb{F}_{q}$, embeds the edge set of $\Gamma_{q, m}$ into its complement in $\Omega^{\{2\}}$. For the proof 
of statement (4), we notice that the definition of $\Gamma_{q, m}$ implies that $\Gamma_{q, m}=\operatorname{Cay}\left(\mathbb{F}_{q}, S_{q, m}\right)$, and so $\Gamma_{q, m}$ is a normal Cayley graph.

\section{Cliques and Colorings of Generalized Paley graphs}

We denote the clique number, the independence number, and the chromatic number of a graph $\Gamma$ by $\omega(\Gamma), \alpha(\Gamma)$, and $\chi(\Gamma)$, respectively. In a vertex-transitive graph $\Gamma$ with $n$ vertices, the existence of a clique $C$ and an independent set $A$ such that $|C||A|=n$ guarantees that $\omega(\Gamma)=|C|$ and $\alpha(\Gamma)=|A|$. We prove this in Lemma 4.2 and in the proof we use the following technical result; see [Cam10, Chapter 3, Theorem 8].

Lemma 4.1. Suppose that $G$ is a transitive group acting on $\Omega$ and $A$ and $B$ are subsets of $\Omega$ such that $|A||B|=|\Omega|$. Then the following are equivalent:

(1) for all $g \in G,|A g \cap B| \geqslant 1$;

(2) for all $g \in G,|A g \cap B|=1$;

(3) for all $g \in G,|A g \cap B| \leqslant 1$.

The next observation will be used in the proof of the next result: if $C$ is a clique and $A$ is an independent set of a graph, then $|C \cap A| \leqslant 1$.

Lemma 4.2. Let $\Gamma$ be a vertex-transitive graph with $n$ vertices, let $C$ be a clique, and let $A$ be an independent set in $\Gamma$ such that $|C||A|=n$. Then $\omega(\Gamma)=|C|$ and $\alpha(\Gamma)=|A|$.

Proof. It suffices to show that $\omega(\Gamma)=|C|$, as the equality $\alpha(\Gamma)=|A|$ will follow by considering the complement graph $\bar{\Gamma}$. Assume by contradiction that there is a clique $D$ in $\Gamma$ such that $|D|>|C|$. Assume without loss of generality that $|D|=|C|+1$. Let $\beta \in D$ be a fixed element, and set $D_{1}=D \backslash\{\beta\}$. Then $D_{1}$ is a clique such that $\left|D_{1}\right|=|C|$. As $D_{1} g$ is a clique for all $g \in \operatorname{Aut}(\Gamma)$ and $A$ is an independence set, $\left|D_{1} g \cap A\right| \leqslant 1$. Since $\left|D_{1}\right||A|=n$, Lemma 4.1 gives that $\left|D_{1} g \cap A\right|=1$ for all $g \in \operatorname{Aut}(\Gamma)$. As $\operatorname{Aut}(\Gamma)$ is transitive on the vertices, there is $g_{0} \in \operatorname{Aut}(\Gamma)$ such that $\beta g_{0} \in A$. Since $\left|D_{1} g_{0} \cap A\right|=1$, we find that $\left|D g_{0} \cap A\right|=2$. Since $D g_{0}$ is a clique and $A$ is an independent set, this is a contradiction. Thus $\omega(\Gamma)=|C|$ as was required.

Using the fact that a generalized Paley graph is a normal Cayley graph, we obtain that the property $\omega(\Gamma)=\chi(\Gamma)$ that appears in Lemma 2.3 follows from a weaker hypothesis.

Lemma 4.3 (Corollary 6.1 .3 of [God]). If $\Gamma$ is a normal Cayley graph such that $\alpha(\Gamma) \omega(\Gamma)$ is equal to the number of vertices of $\Gamma$, then $\omega(\Gamma)=\chi(\Gamma)$.

Lemma 4.3 has an immediate consequence for generalized Paley graphs. 
Corollary 4.4. Set $\Gamma=\Gamma_{q, m}$ such that $m \mid q-1$ and $m \geqslant 2$. The following are equivalent.

(1) $\omega(\Gamma) \alpha(\Gamma)=q$;

(2) $\omega(\Gamma)=\chi(\Gamma)$;

(3) $\omega(\bar{\Gamma}) \alpha(\bar{\Gamma})=q$;

(4) $\omega(\bar{\Gamma})=\chi(\bar{\Gamma})$.

Proof. Since $\Gamma$ is a normal Cayley graph (Corollary [3.3), (1) implies (2) by Lemma 4.3. Suppose that (2) holds and let $C$ be a clique and $\mathcal{P}$ be a coloring such that $|C|=|\mathcal{P}|=k$. Then $\mathcal{P}$ is a $G_{q, m}$-regular partition of the vertex set such that the $G_{q, m}$-regularity is witnessed by $C$. Hence $\mathcal{P}$ is a uniform partition by Lemma 2.1. Thus if $P \in \mathcal{P}$, then $P$ is an independent set such that $|C||P|=q$ and so, by Lemma 4.2, $\omega(\Gamma)=k$ and $\alpha(\Gamma)=|P|=n / k$. Hence (1) is valid. This shows that (1) and (2) are equivalent. Similarly, we obtain that (3) and (4) are equivalent. Since $\omega(\Gamma)=\alpha(\bar{\Gamma})$ and $\alpha(\Gamma)=\omega(\bar{\Gamma})$, (1) is equivalent to (3) and the corollary is valid.

In order to enrich our set of tools to study the clique and chromatic numbers of generalized Paley graphs, we use the $\vartheta$-function, a graph invariant defined by Lovász [Lov79]. The value of $\vartheta(\Gamma)$ for vertextransitive and edge-transitive graphs can be determined by the eigenvalues of the adjacency matrix. As traditional, we let $\lambda_{1}^{\Gamma}$ and $\lambda_{n}^{\Gamma}$ denote the largest and the smallest eigenvalues of $\Gamma$, respectively.

Proposition 4.5 ([Lov79]). Let $\Gamma=(\Omega, E)$ be a graph with vertex set of size $n$. Then the following hold.

(1) $\omega(\Gamma) \leqslant \vartheta(\bar{\Gamma}) \leqslant \chi(\Gamma)$.

(2) If $\Gamma$ is vertex-transitive then $\vartheta(\Gamma) \vartheta(\bar{\Gamma})=n$.

(3) If $\Gamma$ is regular then $\lambda_{1}^{\Gamma}=\partial(\Gamma)$.

(4) If $\Gamma$ is regular and edge-transitive then $\vartheta(\Gamma)=-n \lambda_{n}^{\Gamma} /\left(\lambda_{1}^{\Gamma}-\lambda_{n}^{\Gamma}\right)$.

Using Proposition 4.5, we obtain a necessary condition for the equality $\chi\left(\Gamma_{q, m}\right)=\omega\left(\Gamma_{q, m}\right)$ to hold in vertex-transitive and edge-transitive graphs.

Theorem 4.6. Let $\Gamma$ be a vertex-transitive and edge-transitive graph with $n$ vertices such that $\omega(\Gamma)=\chi(\Gamma)=k$. Then $k-1$ divides $\partial(\Gamma)$ and $\lambda_{n}^{\Gamma}=-\partial \Gamma /(k-1)$.

Proof. Let $\Gamma$ be a graph satisfying the conditions of the theorem. Since $\omega(\Gamma)=\chi(\Gamma)=k$, the first statement of Proposition 4.5 implies that $\omega(\Gamma)=\vartheta(\bar{\Gamma})=\chi(\Gamma)$ and therefore $\vartheta(\bar{\Gamma})=k$. As $\Gamma$ is vertex-transitive, Proposition 4.5 (2) gives $\vartheta(\Gamma)=n / k$. Also, by assumption, $\Gamma$ is regular and therefore we obtain, combining statements (3) and (4) of Proposition 4.5, that

$$
\vartheta(\Gamma)=\frac{n}{k}=\frac{-n \lambda_{n}^{\Gamma}}{\partial(\Gamma)-\lambda_{n}^{\Gamma}} .
$$


Thus $\lambda_{n}^{\Gamma}(1-k)=\partial(\Gamma)$. Therefore $\lambda_{n}^{\Gamma}$ is a rational number. However, since $\lambda_{n}^{\Gamma}$ is an eigenvalue of the adjacency matrix of $\Gamma$, it follows, using the rational root test, that $\lambda_{n}^{\Gamma}$ is an integer. Thus $(k-1)$ divides $\partial(\Gamma)$ and $\lambda_{n}^{\Gamma}=-\partial(\Gamma) /(k-1)$.

Next we state and prove the main result of this section.

Theorem 4.7. Let $p$ be an odd prime and $n \in \mathbb{N}$. Set $q=p^{n}$. Let $m>1$ be such that $2 m \mid q-1$ and set $\Gamma=\Gamma_{q, m}$. Then the following hold.

(1) If $\omega(\Gamma)=\chi(\Gamma)$, then $\omega(\Gamma)=\chi(\Gamma)=p^{t}$, with some $t$ such that $t \mid n$.

(2) If $n$ is a prime and $\omega(\Gamma)=\chi(\Gamma)$, then $m \mid p^{n-1}+p^{n-2}+\cdots+p+1$. Further, in this case, $\omega(\Gamma)=\chi(\Gamma)=p$.

(3) Suppose that $n$ is even. Then $\omega(\Gamma)=\chi(\Gamma)=p^{n / 2}$ if and only if $m \mid\left(p^{n / 2}+1\right)$. In particular, $\omega\left(\Gamma_{p^{2}, m}\right)=\chi\left(\Gamma_{p^{2}, m}\right)$ if and only if $m \mid(p+1)$.

(4) If $n$ is odd and $m$ is even then $\omega(\Gamma) \neq \chi(\Gamma)$.

Proof. (1) Assume that $\omega(\Gamma)=\chi(\Gamma)=k$. By Corollary 3.3 (1), the group $G_{q, m}$ is a vertex-transitive subgroup of $\operatorname{Aut}(\Gamma)$. If $\mathcal{P}$ is a coloring with $k$ colors and $C$ is a clique with $|C|=k$, then $C g$ is a clique for all $g \in G_{q, m}$. Therefore $\mathcal{P}$ is a $G_{q, m}$-regular partition for the vertex set $\mathbb{F}_{q}$ of $\Gamma$ such that the $G_{q, m}$-regularity is witnessed by $C$. Hence Lemma 2.1(1) gives that $k$ divides the number of vertices of $\Gamma$; that is, $k=p^{t}$, with $t<n$. Furthermore, using Theorem 4.6, we have that $p^{t}-1$ divides $\partial(\Gamma)=\left(p^{n}-1\right) / m$, and so $\left(p^{t}-1\right) \mid\left(p^{n}-1\right)$. Elementary argument shows that this implies that $t \mid n$.

(2) Suppose that $n$ is a prime and that $\omega(\Gamma)=\chi(\Gamma)$. Then it follows from (1) that $\omega(\Gamma)=\chi(\Gamma)=p$. Therefore Theorem 4.6 shows that $(p-1) \mid\left(p^{n}-1\right) / m$. As $(p-1)\left(p^{n-1}+p^{n-2}+\cdots+p+1\right)=p^{n}-1$, this implies the assertion.

(3) Assume that $n$ is even. If $\omega(\Gamma)=\chi(\Gamma)=p^{n / 2}$ then we have, by Theorem 4.6, that $\left(p^{n / 2}-1\right) \mid\left(p^{n}-1\right) / m$ which is equivalent to $m \mid\left(p^{n / 2}+1\right)$. Conversely, suppose that $m \mid\left(p^{n / 2}+1\right)$. In this case $\mathbb{F}_{p^{n / 2}}^{*}$ is a subgroup of $S_{p^{n}, m}$, and so the subfield $\mathbb{F}_{p^{n / 2}}$ is a clique in $\Gamma$. This gives that $\omega(\Gamma) \geqslant p^{n / 2}$ and also that $\alpha(\bar{\Gamma}) \geqslant p^{n / 2}$ where $\bar{\Gamma}$ is the complement of $\Gamma$. On the other hand, by Corollary 3.3 , the graph $\Gamma$ can be embedded into $\bar{\Gamma}$, which shows that $\omega(\bar{\Gamma}) \geqslant p^{n / 2}$. By Lemma 4.2, $\omega(\bar{\Gamma})=\alpha(\bar{\Gamma})=p^{n / 2}$, and so $\omega(\Gamma)=\alpha(\Gamma)=p^{n / 2}$. Thus Lemma 4.3 implies that $\omega(\Gamma)=\chi(\Gamma)=p^{n / 2}$.

(4) Suppose now that $n$ is odd and that $\omega(\Gamma)=\chi(\Gamma)=p^{t}$. As $\left(p^{t}-1\right) \mid\left(p^{n}-1\right) / m$, we find that $m \mid\left(p^{n}-1\right) /\left(p^{t}-1\right)$ (which is an integer as $t \mid n)$. As $n / t$ is odd, $\left(p^{n}-1\right) /\left(p^{t}-1\right)$ is a sum of $n / t p$-th powers, and hence it is an odd number, which implies that $m$ is an odd number. Therefore (4) is valid. 


\section{Synchronization of SOME AFFFine Groups}

We apply the results regarding the clique and chromatic numbers of generalized Paley graphs in Section 4 to obtain information about the synchronization property of the 1-dimensional affine groups $G_{q, m}$ for odd $q$. Before stating the first result, recall that a linear group acting on a vector space $V$ is said to be imprimitive if it preserves a direct sum decomposition

$$
V=V_{1} \oplus \cdots \oplus V_{s}
$$

If this is not the case, then the such a linear group is said to be primitive. If $p$ is a prime, then the field $\mathbb{F}_{p^{n}}$ can be considered as an $n$ dimensional vector space over $\mathbb{F}_{p}$ and $Z_{p^{n}, m}$ can be considered as a subgroup of $\mathrm{GL}(n, p)$.

Theorem 5.1. If $Z_{q, m}$ is an imprimitive subgroup of $\mathrm{GL}(n, p)$, then $G_{q, m}$ is non-synchronizing.

Proof. Suppose that $Z_{q, m}$ preserves the direct sum decomposition as in (3). Set, for $i \in\{1, \ldots, s\}$ and $v \in V_{i}$,

$$
P_{i, v}=\left\{v_{1}+\cdots+v_{n} \in V \mid v_{i}=v\right\}
$$

and consider the partition $\mathcal{P}_{i}=\left\{P_{i, v} \mid v \in V_{i}\right\}$. Then consider the set of partitions $\Sigma=\left\{\mathcal{P}_{1}, \ldots, \mathcal{P}_{s}\right\}$. It follows from the imprimitivity of $Z_{q, m}$ and the definition of cartesian decompositions that $\Sigma$ is a cartesian decomposition which is preserved by $G_{q, m}$. Thus, by Lemma 2.2, we conclude that $G_{q, m}$ is a non-synchronizing group.

Theorem 5.2. Suppose that $q=p^{n}$ where $p$ is an odd prime and let $m$ be a divisor of $q-1$. Set $\bar{m}$ as in equation (1).

(1) If $\omega\left(\Gamma_{q, \bar{m}}\right)=\chi\left(\Gamma_{q, \bar{m}}\right)$ then $G_{q, m}$ is non-synchronizing.

(2) If $m \in\{2,3\}$, then $G_{q, m}$ is non-synchronizing if and only if $\omega\left(\Gamma_{q, m}\right)=\chi\left(\Gamma_{q, m}\right)$.

(3) Suppose that $n$ is a prime and that $m \in\{2,3\}$. If

$$
m \nmid p^{n-1}+p^{n-2}+\cdots+p+1,
$$

then $G_{q, m}$ is synchronizing.

(4) If $n$ is even and $\operatorname{gcd}\left(m, p^{n / 2}+1\right) \neq 1$, then $G_{q, m}$ is non-synchronizing.

(5) If $n$ is odd, then $G_{q, 2}$ is synchronizing.

(6) The group $G_{p^{2}, 2}$ is always non-synchronizing, while $G_{p^{2}, 3}$ is nonsynchronizing if and only if $m \mid p+1$.

Proof. (1) By Lemma 3.2, the generalized Paley graph $\Gamma_{q, \bar{m}}$ is $G_{q, m^{-}}$ invariant. If $\omega\left(\Gamma_{q, \bar{m}}\right)=\chi\left(\Gamma_{q, \bar{m}}\right)$, then Lemma 2.3 shows that $G_{q, m}$ is non-synchronizing.

(2) Suppose that $m \in\{2,3\}$. In this case $\bar{m}=m$. If $\Gamma$ is a $G_{q, m^{-}}$ invariant graph, then, as $G_{q, m}$ has 2 or 3 orbits in $\Omega^{\{2\}}$, Lemma 3.2 
shows that $\Gamma$ is isomorphic either to $\Gamma_{q, m}$ or to the complement $\bar{\Gamma}_{q, m}$. On the other hand, Corollary 4.4 shows that $\omega\left(\Gamma_{q, m}\right)=\chi\left(\Gamma_{q, m}\right)$ if and only if $\omega\left(\bar{\Gamma}_{q, m}\right)=\chi\left(\bar{\Gamma}_{q, m}\right)$. This implies that $\omega(\Gamma)=\chi(\Gamma)$ if and only if $\omega\left(\Gamma_{q, m}\right)=\chi\left(\Gamma_{q, m}\right)$.

(3) If $n$ is prime, $m \in\{2,3\}$, and $m \nmid p^{n-1}+p^{n-2}+\cdots+p+1$, then Theorem $4.7(2)$, gives that $\omega\left(\Gamma_{q, m}\right) \neq \chi\left(\Gamma_{q, m}\right)$. Thus part (1) shows that $G_{q, m}$ is synchronizing.

(4) Suppose that $n$ is even and $d=\operatorname{gcd}\left(m, p^{n / 2}+1\right) \neq 1$. Since $d \mid p^{n / 2}+1$, Theorem 4.7(3) gives that $\omega\left(\Gamma_{q, d}\right)=\chi\left(\Gamma_{q, d}\right)$, and hence, by part (1), $G_{q, d}$ is non-synchronizing. On the other hand, as $d \mid m$, we have that $G_{q, m} \leqslant G_{q, d}$, and hence $G_{q, m}$ is non-synchronizing also.

(5) This follows combining part (2) with Theorem 4.7(4).

(6) Part (4) implies that $G_{p^{2}, 2}$ is always non-synchronizing. By part (2), $G_{p^{2}, 3}$ is non-synchronizing if and only if $\omega\left(\Gamma_{p^{2}, 3}\right)=\chi\left(\Gamma_{p^{2}, 3}\right)$ which happens if and only if $m \mid p+1$; see Theorem 4.7)(3).

\section{REFERENCES}

[A08] João Araújo. A group theoretical approach to synchronizing automata and the Černý problem. Unpublished notes, 2008.

[AS06] Fredrick Arnold and Benjamin Steinberg. Synchronizing groups and automata. Theoret. Comput. Sci., 359(1-3):101-110, 2006.

[BDR88] I. Broere, D. Döman, and J. N. Ridley. The clique numbers and chromatic numbers of certain Paley graphs. Quaestiones Math., 11(1):91-93, 1988.

[Cam99] Peter J. Cameron. Permutation groups, volume 45 of London Mathematical Society Student Texts. Cambridge University Press, Cambridge, 1999.

[Cam10] Peter J. Cameron. Synchronization http://www.maths.qmul.ac.uk/ pjc/LTCC-2010-intensive3/, 2010.

[Čer64] J. Cerný. Poznámka k homogénnym eksperimentom s konecnými avtomatami. Mat.-Fyz. Cas. Solvensk. Akad., 14:208-216, 1964.

[DM96] John D. Dixon and Brian Mortimer. Permutation groups, volume 163 of Graduate Texts in Mathematics. Springer-Verlag, New York, 1996.

[God] Chris Godsil. Interesting graphs and their colourings. University of Waterloo, 2004.

[Lov79] László Lovász. On the Shannon capacity of a graph. IEEE Trans. Inform. Theory, 25(1):1-7, 1979.

[LP09] Tian Khoon Lim and Cheryl E. Praeger. On generalized Paley graphs and their automorphism groups. Michigan Math. J., 58(1):293-308, 2009.

[Mik] Mike (mathoverflow.net/users/1579). Cliques, Paley graphs and quadratic residues. MathOverflow. http://mathoverflow.net/questions/48591 (version: 2010-12-07).

[Neu09] Peter M. Neumann. Primitive permutation groups and their sectionregular partitions. Michigan Math. J., 58(1):309-322, 2009.

[Pal33] R. E. A. C. Paley. On Orthogonal Matrices. J. Math. Phys. Inst. Tech., 12:311-320, 1933.

[PBS03] Cheryl E. Praeger, Robert W. Baddeley, and Csaba Schneider. Transitive simple subgroups of wreath products in product action. 2003. 
[Sho92] M. W. Short. The primitive soluble permutation groups of degree less than 256, volume 1519 of Lecture Notes in Mathematics. Springer-Verlag, Berlin, 1992.

(Schneider) Departamento de Matemática, Instituto de Cincias Exatas, Universidade Federal de Minas Gerais, Av. Antônio Carlos 6627, 31270-901, Belo Horizonte, MG, Brazil

(Silva) Departamento de Matemtica, Faculdade de Cincias da Universidade de Lisboa, Campo Grande, 1749-016 Lisboa, Portugal 